\title{
PESQUISA DE YERSINIA PESTIS EM ROEDORES E OUTROS PEQUENOS MAMÍFEROS NOS FOCOS PESTOSOS DO NORDESTE DO BRASIL NO PERÍODO 1966 A 1982
}

\author{
Alzira Maria Paiva de Almeida * \\ Darci Pascoal Brasil * \\ Francisco Gomes de Carvalho ** \\ Célio Rodrigues de Almeida ***
}

\begin{abstract}
ALMEIDA, A.M.P. de et al. Pesquisa de Yersinia pestis em roedores e outros pequenos mamíferos nos focos pestosos do Nordeste do Brasil no período 1966 a 1982. Rev. Saúde públ., S. Paulo, 21:265-7, 1987.

RESUMO: Foi feita análise da metodologia empregada e dos resultados alcançados em pesquisa de Yersinia pestis, em material de 24.703 roedores e outros pequenos mamíferos oriundos dos focos pestosos do Nordeste do Brasil, no período de 1966 a 1982. Concluiu-se ser necessário haver maior rapidez na realização dos exames para que os dados obtidos sejam convenientemente aplicados nas atividades de vigilância e controle da peste.
\end{abstract}

UNITERMOS: Yersinia pestis, isolamento. Roedores, microbiologia. Peste, prevenção e controle. Reservatório de doenças.

A peste ainda representa problema epidemiológico em muitos países do mundo. Concentrada entre os roedores dos focos naturais de onde não se pode erradicar, vem produzindo casos esporádicos ou surtos epidêmicos ocasionais entre as populações humanas. Cada foco tem características peculiares, com seu próprio ciclo epidemiológico, alternando períodos de atividade com periodos de quiescência. Em condições especiais a infecção pode se expandir e atingir novas áreas. Em vista do potencial de rápida expansão e alta letalidade, fazse necessária a manutenção de um sistema de vigilância e controle permanente a fim de se conhecer a extensão da infecção a cada momento, entre os roedores, outros mamíferos e seus ectoparasitos ${ }^{8}$. A vigilância da peste no Brasil está baseada no rastreamento da infecção nos campos através da captura de roedores susceptíveis, coleta dos pulicídeos vetores e pesquisa do bacilo nestas fontes e/ou por inquéritos sorológicos entre os roedores e outros pequenos mamíferos e carnívoros domésticos. Neste trabalho analisa-se a pesquisa da Yersinia pestis em 24.703 roedores e outros pequenos mamíferos oriundos dos focos de peste do Nordeste do Brasil, no período de 1966 a 1982.

Em 17.745 animais as análises para identificação da Y.pestis foram efetuadas logo após a necrópsia, seguindo-se a metodologia de Karimi ${ }^{5}$, e em 6.958 animais foi adotado o processo de conservação dos baços no meio de Cary-Blair recomendado por Cavanaugh e col. ${ }^{3}$ para o transporte de espécimes contendo Y.pestis e análise posterior num Laboratório Central.

\section{RESULTADOS E COMENTÁRIOS}

A Y.pestis foi encontrada em $416(2,34 \%)$ animais dos 17.745 cujo material foi analisado por cultivos e/ou inoculações de triturados de vísceras logo após a coleta e em $55(0,79 \%)$ dos 6.958 submetidos a conservação no meio de Cary - Blair. Estes resultados são significativamente diferentes pelo teste do qui-quadrado ${ }^{4}$ ao nível de rejeição de $1 \%$. A Tabela mostra a distribuição dos animais examinados e animais infectados por espécie e por foco.

Comparando os resultados obtidos no foco da Chapada do Araripe nos dois períodos analisados pelo teste do qui-quadrado, verificamos uma diferença significativa ao nível de rejeição de $1 \%$. No período de 1966 a 1974, a análise imediata dos triturados de vísceras dos roedores possibilitou acompanhar os rastilhos epizoóticos pela intensificação das capturas nos locais da epizootia e obter maior número de animais infectados. A substituição deste procedimento pela conservação dos baços dos roedores em Cary-Blair, para transporte e análise posterior, não permitiu o acompanhamento dos rastilhos epizoóticos no período seguinte. As atividades de captura dos roedores, que eram orientadas pelos resultados obtidos diariamente no laboratório, passaram a obedecer um roteiro estabelecido anualmente. Além das modificações introduzidas na metodologia de trabalho, outro fator que pode ter contribuído para a redução dos animais infectados do Foco da Chapada do Araripe, no período de 1975 a 1982, foi o esgotamento da fauna ro-

* Laboratório de Peste do Centro de Pesquisas Aggeu Magalhães - Caixa Postal 7472 - 50000 - Recife, PE - Brasil

** Laboratório de Peste - 56230 - Exu, PE - Brasil.

*** Bolsista do CNPq, junto ao Laboratório de Peste - Recife, PE - Brasil. 
Pesquisa da Yérsinia pestis em roedores e outros pequenos mamíferos nos focos do Nordeste do Brasil, no período de 1966 a 1982

\begin{tabular}{|c|c|c|c|c|c|c|c|c|}
\hline \multirow[b]{2}{*}{ Espécies } & \multicolumn{7}{|c|}{ FOCOS } & \multirow[b]{2}{*}{ Totais } \\
\hline & $\begin{array}{l}\text { Chapada do } \\
\text { 1966-1974* }\end{array}$ & $\begin{array}{l}\text { Arripe/PE } \\
1975-1982\end{array}$ & $\begin{array}{c}\text { Triunfo/PE } \\
1975-1982\end{array}$ & $\begin{array}{l}\text { Planalto de } \\
\text { Agreste/PE } \\
\text { 1975-1982* }\end{array}$ & $\begin{array}{l}\text { a Borborema } \\
\text { Encosta Leste/PB } \\
1975-1982\end{array}$ & $\begin{array}{c}\text { Norte do } \\
\text { Ceará } \\
1976-1982 \\
\end{array}$ & $\begin{array}{c}\text { Planalto } \\
\text { Oriental/BA } \\
1975-1979 \\
\end{array}$ & \\
\hline & Exam. (+) & Exam.(+) & Exam.(+) & Exam. $(+)$ & Exam.(+) & Exam. $(+)$ & Exam.(+) & Exam. $(+)$ \\
\hline Rattus $r$. alexandrinus & $1124(18)$ & 149 & $56(1)$ & 81 & 75 & 16 & - & $1501(19)$ \\
\hline Rattus r. frugivorus & $821(12)$ & 186 & 6 & 184 & 70 & 17 & - & $1284(12)$ \\
\hline Rattus r. rattus & 12 & 1 & 2 & - & 53 & 9 & - & 77 \\
\hline Mus musculus brevirostris & - & 3 & 8 & 14 & 119 & - & - & 144 \\
\hline Akodon $a$. arviculoides & - & - & - & 4 & - & - & - & 4 \\
\hline Calomys callosus & $439(2)$ & 49 & - & 244 & 149 & $1(1)$ & - & $882(3)$ \\
\hline Oryzomys eliurus & $394(4)$ & $37(1)$ & 5 & 451 & 23(1) & - & 1 & $911(6)$ \\
\hline Oryzomys subflavus & $575(18)$ & $114(2)$ & $321(5)$ & $810(1)$ & $510(1)$ & 3 & - & $2333(27)$ \\
\hline Bolomys lasiurus** & $7578(334)$ & $1311(15)$ & $396(9)^{* * *}$ & $2667(11)$ & $1404(7)$ & $592(5)$ & 6 & $13954(381)$ \\
\hline Wiedomys pyrrhorinos & 78 & 1 & - & 2 & 6 & - & - & 87 \\
\hline Holochilus scureus & - & - & - & 1 & 18 & 5 & - & 24 \\
\hline Oxymycterus angularis & - & - & - & 10 & - & - & - & 10 \\
\hline Trichomys apereoides & $547(9)$ & $130(4)$ & 14 & 13 & 14 & 1 & - & $719(13)$ \\
\hline Galea spixii wellsi & $1137(6)$ & 503 & 79 & 101 & 132 & 10 & 1 & 1963(6) \\
\hline Kerodon rupestris & 117 & - & - & - & - & - & - & 117 \\
\hline Marmosa Karimi & 10 & - & - & - & - & - & - & 10 \\
\hline Monodelphis $d$. domestica & $220(1)$ & $102(1)$ & 49 & 81 & 5 & - & 1 & $458(2)$ \\
\hline Didelphis p. paraguaiensis & 24 & 5 & - & 6 & - & 1 & - & 36 \\
\hline Sem informação & - & 76 & 20 & - & 79 & $1(1)$ & $13(1)$ & $189(2)$ \\
\hline \multirow{2}{*}{$\begin{array}{l}\text { Total } \\
\%\left(\frac{\text { infectados }}{\text { examinados }}\right.\end{array}$} & $13076(404)$ & $2667(23)$ & $956(15)$ & $4669(12)$ & $2657(9)$ & $656(7)$ & $22(1)$ & $24703(471)$ \\
\hline & 3,09 & 0,86 & 1,57 & 0,26 & 0,34 & 1,07 & 4,54 & 1,90 \\
\hline
\end{tabular}

* Amostras examinadas após a colheita, nos demais foi usado meio de conservação e transporte.

** (Zygodontomys lasiurus pixuna).

*** Incluída uma positivação de 1969 em 4 animais examinados.

dentia, refletida inclusive na diminuição da quantidade de animais examinados. As populações de roedores dizimadas pelas epizootias sucessivas dos anos anteriores não conseguiram se refazer durante a prolongada estiagem que ocorreu na região entre o fim da década de 70 e início de 80. A diminuição da incidência da peste humana nesse foco também foi observada durante esse período ${ }^{1}$. Nas duas áreas estudadas do foco do Planalto da Borborema, no período de 1975-1982, empregando metodologia diferente (exame de material dos roedores do Agreste/PE logo após a colheita e conservação do material dos roedores da Encosta Leste/PB no meio de Cary-Blair), os resultados obtidos são estatísticamente comparáveis pelo teste do qui-quadrado ao nível de rejeição de 1\%. Entretanto, quando se comparam os dados obtidos no mesmo período no foco da Chapada do Araripe, em Triunfo e na Encosta Leste/PB, apesar de haver sido empregada metodologia semelhante (conservação do material em Cary-Blair) os resultados são diferentes estatísticamente pelo teste do qui-quadra- do ao nível de rejeição de $1 \%$. Estes resultados mostram que cada foco tem seu próprio ciclo epidemiológico e são independentes no tempo e no espaço².

Quanto aos resultados obtidos nos focos Norte do Ceará e Planalto Oriental/BA, ressaltamos que os animais infectados foram na maioria obtidos por ocasião de inquéritos epidemiológicos durante surtos de peste humana, enquanto que nos focos de Triunfo e Planalto da Borborema os animais foram obtidos pela procura de atividade pestosa nos campos, seguindo um itinerário estabelecido anualmente. Por estes resultados concluimos que apesar da eficácia do meio de Cary-Blair na manutenção da Yersinia pestis, mostrada por diversos autores, $3,6,8$ a demora na realização dos exames foge ao seu objetivo principal que é a detecção precoce de atividade pestosa e o acompanhamento dos rastilhos epizoóticos. Por conseguinte, é necessário que se agilize o envio das amostras aos laboratórios de diagnóstico, assim como o seu exame nesses laboratórios. 
ALMEIDA, A.M.P. de et al. [Detection of Yersinia pestis in rodents and other small mammals in the northeast of Brazil during the period from 1966 to 1982]. Rev. Saúde públ., S. Paulo, 21:265-7, 1987.

ABSTRACT: The analysis of the methods employed and the results obtained in the research into Yersinia pestis in 24.703 rodents and other small mammals from plague foci in the Northeast of Brazil during the period from 1966 to 1982 , shows that the examinations should be carried out more guickly, to make prompter use of the data obtained in the activities of plague surveillance and control possible.

UNITERMS: Yersinia pestis, isolation. Rodents, microbiology. Plague, prevention and control. Disease reservoirs.

\section{REFERÊNCIAS BIBLIOGRÁFICAS}

1. ALMEIDA, A.M.P.; BRASIL, D.P.; CARVALHO, F.G.; ALMEIDA, C.R. Isolamento de Yersinia pestis nos focos pestosos do Norteste do Brasil no período de 1966 a 1982. Rev. Inst. Med. trop. S.Paulo, 27: 207-18, 1985.

2. BALTAZARD, M. Viagem de estudo ao Brasil para a organização de um projeto de pesquisas sobre a peste. Rev. bras. Malar., 20: 335-6, 1968.

3. CAVANAUGH, D.C.; VIVONA, S.; DO-VAN-QUY; GIBSON, F.L.; DEUBER, G.L.; RUST Jr., J.H. A transport medium for specimens containing P.pestis. Bull. Wld Hlth Org., 37: 455-9, 1967.

4. GOMES, F.P. Curso de estatística experimental. 6a ed. São Paulo, Livraria Nobel, 1976.

5. KARIMI, Y. Diagnostique rapide de l'infection pesteuse au Laboratoire. Bull. Soc. Path. exot., 1: 45-8, 1978.
6. MELLO, M.T.; PARACAMPO, H.; SANTOS, D.A. Emprego do meio de Cary-Blair para conservação e transporte de material suspeito de peste em zonas rurais. Rev.bras.Malar., 21: 767-73, 1969.

7. SURVEILLANCE de la peste et lutte anti-pesteuse. Cron.Org.mund.Salud, 34: 152-6, 1980.

8. ZEBRAL, A.A. \& COSTA, G.A. Eficácia de alguns meios de cultura na conservação de amostras de Yersinia pestis. Rev. bras. Malar., 31: 5-17, 1969.

Recebido para publicação em 20/10/1986

Reapresentado em 16/3/1987

Aprovado para publicação em 19/3/1987 